\title{
Interstitial cells of Cajal (ICC)-like-c-Kit positive cells are involved in gastritis and carcinogenesis in human stomach
}

\author{
ROHYUN SUNG ${ }^{1 *}$, YOUNG CHUL KIM ${ }^{2 *}$, HYO-YUNG YUN ${ }^{3 *}$, WOONG CHOI ${ }^{4,9}$, \\ HUN SIK KIM ${ }^{4}$, HEON KIM ${ }^{5}$, KWANG JU LEE ${ }^{1}$, RA YOUNG YOU ${ }^{2}$, SEON MEE PARK ${ }^{6}$, \\ SEI JIN YOUN ${ }^{6}$, MI-JUNG KIM ${ }^{7}$, WON SEOP KIM ${ }^{7}$, YOUNG-JIN SONG ${ }^{3}$, \\ SEOK-YONG KIM ${ }^{8}$, WEN-XIE XU ${ }^{10}$ and SANG JIN LEE ${ }^{2}$
}

\begin{abstract}
Departments of ${ }^{1}$ Pathology, ${ }^{2}$ Physiology, ${ }^{3}$ Surgery, ${ }^{4}$ Pharmacology, ${ }^{5}$ Preventive Medicine, ${ }^{6}$ Internal Medicine, ${ }^{7}$ Pediatrics and ${ }^{8}$ Microbiology, Chungbuk National University, College of Medicine, Heungduk-gu, Cheongju;

${ }^{9}$ BK21, Chungbuk National University, School of Medicine, Cheongju, Chungbuk, Republic of Korea;

${ }^{10}$ Department of Physiology, College of Medicine, Shanghai Jiaotong University, Shanghai, P.R. China
\end{abstract}

Received January 28, 2011; Accepted March 15, 2011

DOI: 10.3892/or.2011.1293

\begin{abstract}
This study was executed to prove the existence of c-Kit-positive interstitial cells of Cajal (ICC)-like cells [c-Kit (+) ICC-like cells] and their possible role associated with gastric inflammation and/or carcinogenesis in human gastric mucosa. c-Kit (+) ICC-like cells were observed throughout all the layers of the gastric fundus along the greater curvature. Dense fusiform cell bodies with many processes were found in each layer. We also studied the c-Kit-positive immunoreactivity distribution pattern in the mucosa. c-Kit $(+)$ cells were found mainly around the epithelial repair zone of the normal gastric fundus/corpus and of the fundus/corpus with non-metaplastic chronic gastritis. Notably, they were found attached to the epithelia of the repair zone in non-metaplastic chronic gastritis. In chronic gastritis with intestinal metaplasia, they were found scattered everywhere in the stroma of the gastric mucosa and did not attach to the metaplastic epithelium. We found c-Kit (+) ICC-like cells in human mucosa. They were present mainly in the stroma around the repair zone of the glands in chronic gastritis as well as in normal mucosa, whereas they seemed to
\end{abstract}

Correspondence to: Professor Hyo-Yung Yun, Department of Surgery, Chungbuk National University, College of Medicine, 12 Gaeshin-Dong, Heungduk-Gu, Cheongju 361-763, Republic of Korea

E-mail: yunhyo@chungbuk.ac.kr

Professor Young Chul Kim, Department of Physiology, Chungbuk National University, College of Medicine, 12 Gaeshin-Dong, Heungduk-Gu, Cheongju, Chungbuk 361-763, Republic of Korea

E-mail: physiokyc@chungbuk.ac.kr

*Contributed equally

Key words: human gastric mucosa, c-Kit, interstitial cells of Cajal, gastritis, atrophy, intestinal metaplasia, adenocarcinoma redistribute over the whole mucosa in gastritis with intestinal metaplasia. These cells around the repair zone were found to be tightly attached to epithelial cells, but not to metaplastic epithelial cells. Thus, c-Kit (+) ICC-like cells appear to have a role in the epithelial recovery process and may be associated with carcinogenesis of human gastric mucosa.

\section{Introduction}

In 1911, interstitial cells of Cajal (ICC) were described in the gastrointestinal (GI) tract by Cajal (1). ICC are now believed to be an essential regulator of GI digestive motility. Many studies of the past ten years indicate that ICC are pacemaker cells that generate spontaneous electrical activities known as slow waves of the gut, which spread passively via gap junctions to neighboring GI smooth muscle cells and produce spontaneous contractions such as peristalsis $(2,3)$.

ICC have been observed from the esophagus to the anus in a wide variety of species including the human (4-7). To date, at least two types of ICC have been identified in the GI tract: one is ICC located in the myenteric region (ICC-MY) and the other is ICC of the intramusculature (ICC-IM) distributed deep in the muscular plexus through the muscle layers (8). The regenerative pacemaker potential formed from the ICC-MY network connected by gap junctions is electrically coupled to the smooth muscles for harmonious motility (8). Meanwhile, ICC-IM are also believed to be involved in the regulation of neurotransmission (9) even though there are some controversies (10). Since ICC play an important role in the coordination of GI motility, structural destruction has been implicated in many GI motility diseases (11).

Although studies on ICC have mostly focused on their role as a pacemaker and neurological regulator, some ICC have been reported to be a precancerous state for gastrointestinal stromal tumors (GISTs) (12). Notably, studies have shown the ICC have c-Kit-positive immunoreactivity in the microvilli of the stomach and small intestine $(1,13,14)$. These findings indicate the possible existence of ICC other than in the 
musculature. However, the existence of ICC in the mucosa and their functional role have not yet been established (14).

ICC are myoid cells of mesenchymal origin (15) and normally express the proto-oncogene c-Kit. Therefore, antibodies against c-Kit are useful for the identification of ICC $(2,3)$. c-Kit is a transmembrane tyrosine kinase receptor (RTK) and its activation by the stem cell factor (SCF) is essential for survival, maintenance of the phenotype and pacemaking activity of ICC (4). Apart from ICC, however, c-Kit and/or SCF are also expressed in a variety of normal tissues such as placenta and lung (16) and also in gastric carcinoma cell lines (17).

The activation of c-Kit by mutations has been shown in a number of human malignancies such as human gastric carcinomas (17), GIST (18), and colorectal cancer (19). In particular, several risk factors and/or steps in carcinogenesis in the stomach have been reported. These include helicobacter pylori (H. pylori) infection, chronic gastritis, chronic atrophic gastritis, intestinal metaplasia (IM) and dysplasia (20). Furthermore, strong evidence that gastric cancer originates from mesenchymal stem cells (MSCs), bone-marrow-derived sources and transformation of stem cells was noted in the human stomach (21). In the GI tract, ICC, one type of MSCs, express c-Kit and its alterations are reported to induce a change in their cell type (22). Furthermore, tumor growth is also dependent on the tyrosine kinase c-Kit product (CD117). Therefore, ICC exhibit diverse abilities and may be associated with carcinogenesis in the gastric mucosa. However, no gastric mucosal cells except mast cells are reported to have c-Kit expression (23). In fact, ICC are also present in the submucosal plexus, muscularis mucosa and mucosa (1,22-25).

Moreover, it was suggested that the muscularis mucosa also has a pacemaker mechanism (26). Also, many structures within villi, the muscularis mucosa and submucosa are closely related (27). Therefore, it is strongly suggested that ICC are present in the submucosal plexus, muscularis mucosa and even in mucosa and that they play a physiological role in the harmonious movement of mucosa and villi and also for pathologic changes in the GI tract. Previously, it was suggested that ICC also have close contact with several types of immunocytes such as eosinophils, basophils and macrophages (25). In addition, structural changes in ICC have also been reported to be associated with certain inflammation-induced motility disorders $(25,28)$.

Based on recent studies on the function and distribution of ICC, we hypothesized that c-Kit (+) ICC-like cells do exist and play an important pathophysiological role in gastric mucosa. Also, we found that c-Kit (+) ICC-like cells of the human gastric mucosa had uniform distribution patterns according to the stage of diseases; from normal stomach to gastritis, IM and eventually adenocarcinoma. Therefore, we designed this study to investigate whether mucosa expresses c-Kit immunoreactivity and whether c-Kit (+) ICC-like cells have a special role in inflammation and carcinogenesis in human gastric mucosa.

\section{Materials and methods}

Tissue preparation. Human gastric fundus and corpus samples from both the greater and lesser curvature were obtained from 14 gastric cancer patients who underwent subtotal gastrectomy

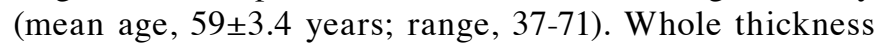

samples were taken from the gastric wall of each patient, in particular from the fundus $(n=4)$ and corpus $(n=9)$ of the greater curvature, and the corpus of the lesser curvature $(n=1)$. Specimens (14 cases) were extracted immediately after the surgical operation from macroscopically normal tissue far from the neoplastic area. All patients provided written informed consent, and the experimental protocol using human stomach was also approved by the Institutional Review Board for Clinical Research, Chungbuk National University, School of Medicine, Korea.

In Krebs-Ringer bicarbonate (KRB) solution, specimens were pinned on a Sylgard plate in order to maintain the original shape and length. Connective tissues were then removed and cut and pinned to rectangular shape $(2 \mathrm{~cm} \times 3 \mathrm{~cm}$, width and length). Finally, these gastric tissues were fixed in $4 \%$ paraformaldehyde in $0.1 \mathrm{M}$ sodium phosphate buffer for $24 \mathrm{~h}$ at room temperature. Two longitudinal sections, $3 \mathrm{~cm}$ in length and $3 \mathrm{~mm}$ in thickness, were taken from each fixed tissue. The sections were processed on a computerized tissue processor (Tissue-Tek VIP; Sakura Finetek, Torrance, CA, USA) and embedded in paraffin (Paraplast Medium; Leica Biosystems, Maarn, The Netherlands) on a tissue embedding console system (Tissue-Tek TEC; Sakura Finetek). Five formalin-fixed paraffin tissue blocks were also retrieved from the archive of the Pathology Department at Chungbuk National University Hospital. They consisted of one tissue block from the normal corpus of the stomach resected using a Whipple operation for pancreatic adenocarcinoma and four tissue blocks from gastric adenocarcinoma resected at subtotal or total gastrectomy.

All paraffin-embedded tissue blocks were sectioned $(4 \mu \mathrm{m})$ with a microtome, and slides were prepared. Paraffin was removed from the slides with xylene treatment. The sections were rehydrated with an ethanol series and stained with hematoxylin and eosin $(\mathrm{H} \& \mathrm{E})$. Final stages of processing were performed with an automated slide stainer (Tissue-Tek Prisma; Sakura Finetek) and an automated coverslipper (Tissue-Tek Glas; Sakura Finetek).

Immunohistochemical labeling of ICC by $c$-Kit. Sections were cut at $4 \mu \mathrm{m}$ from paraffin-embedded gastric tissues and mounted on positively charged slides (Superfrost Plus; VWR International, West Chester, PA, USA). Simultaneous deparaffinization and antigen retrieval prior to immunostaining were accomplished on an automated PT module (Lab Vision, Fremont, CA, USA). Immunohistochemistry was conducting using an automated immunostainer (Autostainer 360; Lab Vision) according to the manufacturer's protocol. Peroxidase staining was carried out using UltraVision LP Detection System HRP Polymer \& DAB Plus Chromogen (Thermo Fisher Scientific, Fremont, CA, USA). Briefly the sections were incubated in Hydrogen Peroxide Block for $10 \mathrm{~min}$ to reduce nonspecific background staining due to endogenous peroxidase. After washing in phosphate buffered saline (PBS) plus Tween-20 (20X) (ScyTek Laboratories, Logan, UT, USA), they were incubated with Ultra $\mathrm{V}$ Block for $5 \mathrm{~min}$ at room temperature to block nonspecific binding. The sections were then incubated with polyclonal rabbit anti-human CD117 (Dako, Carpinteria, CA, USA) at a dilution of 1:400 and monoclonal mouse anti-human mast cell tryptase (clone AA1; Dako, Glostrup, Denmark) at a dilution of 1:200 for 
A
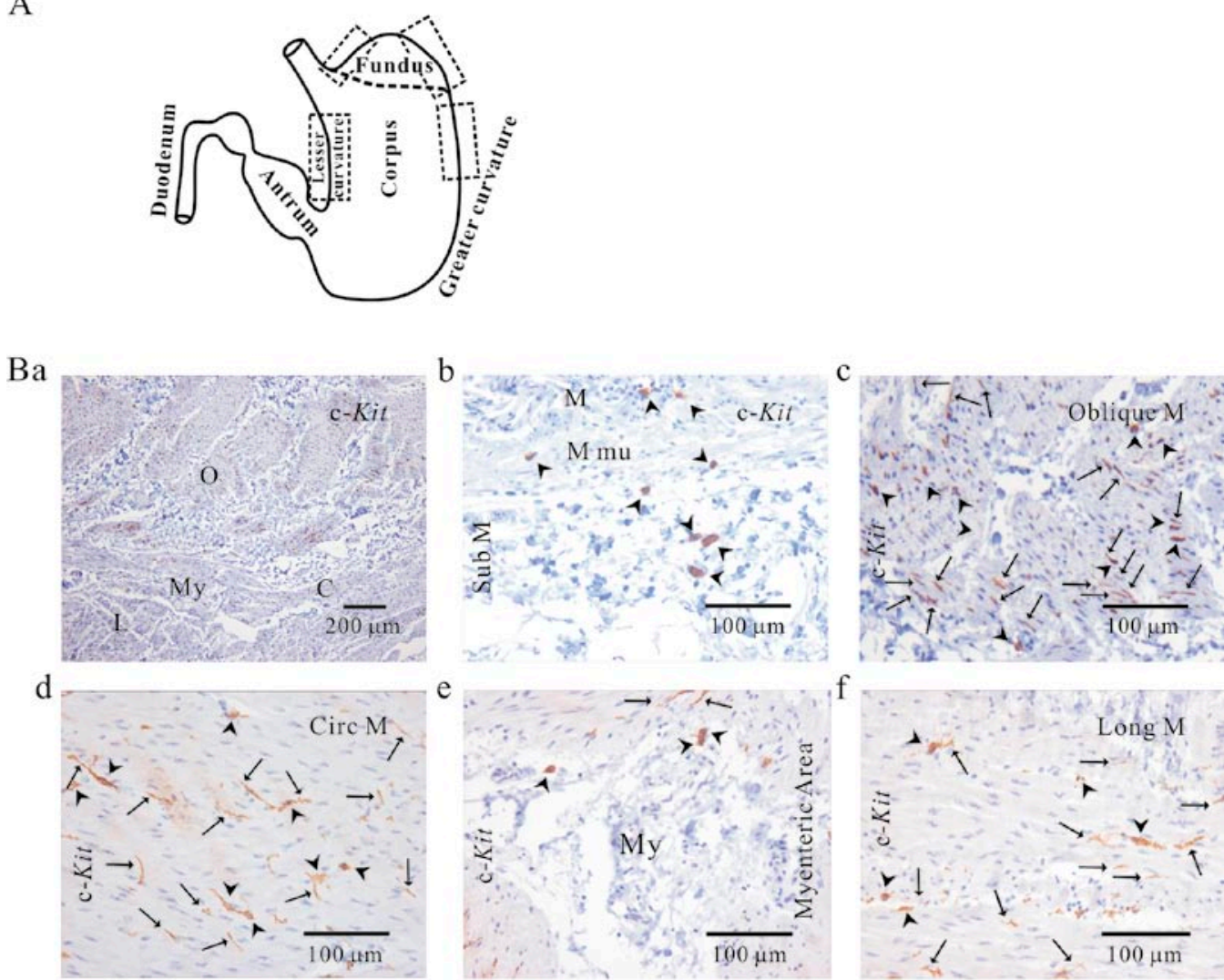

Figure 1. Distribution of interstitial cells of Cajal (ICC) in human normal stomach. Various portions of human stomach were used (A). c-Kit (+) ICCs were observed from the mucosa to the longitudinal muscle in the greater curvature of the fundus (Bb-f). Dark brown long spindle-like cell bodies (arrow heads) and multiple processes (arrows) are shown. M mu, musclaris mucosa; Sub M, submucosa; O, oblique muscle; C, circular muscle; My, myenteric region; and L, longitudinal muscle.

$40 \mathrm{~min}$ at room temperature. After washing in PBS, they were incubated at room temperature with primary antibody enhancer, followed by washes in PBS and incubation with the HRP polymer for $15 \mathrm{~min}$ at room temperature. Sections were then washed in PBS and followed by staining with DAB Plus chromogen and substrate. Counterstaining was performed with hematoxylin. Negative controls were performed by omitting the primary antibodies or by substituting them with a non-immune serum in order to check the specificity of the immunostaining. All sections for histologic and immunohistochemical analysis were examined using a microscope (BX50; Olympus Corporation) and images were captured with an attached camera (ProgRes C14; Jenoptik, Jena, Germany) operated with CapturePro software (Jenoptik).

Solution and drugs. The KRB solution $\left(\mathrm{CO}_{2} /\right.$ bicarbonatebuffered Tyrode's solution) contained (in $\mathrm{mM}$ ): $\mathrm{NaCl} 122$, $\mathrm{KCl} 4.7, \mathrm{MgCl}_{2} 1, \mathrm{CaCl}_{2} 2, \mathrm{NaHCO}_{3} 15, \mathrm{KH}_{2} \mathrm{PO}_{4} 0.93$, and glucose $11\left(\mathrm{pH} 7.3-7.4\right.$, bubbled with $\left.5 \% \mathrm{CO}_{2} / 95 \% \mathrm{O}_{2}\right)$. PBS was purchased from ScyTek Laboratories. All drugs used in this study were purchased from Dako (anti-human c-Kit, anti-human mast cell tryptase) and Sigma.

\section{Results}

The distribution of ICC in human gastric fundus. Gastric tissues were obtained from a patient with gastric cancer and pancreatic cancer who underwent subtotal gastrectomy and a Whipple operation, respectively. Using these samples, we studied the distribution of c-Kit (+) ICC-like cells in normal gastric fundus/corpus. As shown in Fig. 1A, we obtained tissue samples from the gastric fundus and corpus of the greater curvature (GC) and lesser curvature (LC). In every case, we initially identified structures of the stomach using H\&E staining. The oblique, circular and longitudinal muscle layers from the fundus of GC are noted in Fig. 1Ba. The oblique muscle was prominent in the gastric fundus (Fig. 1Ba and c). We previously observed c-Kit-positive immunoreactivity in all of the layers of the GC and LC of the fundus and corpus (29). Long spindle-like (fusiform) cell bodies with multiple processes were noted in the oblique, circular and longitudinal muscle layers, respectively (Fig. $1 \mathrm{Bc}, \mathrm{d}$ and $\mathrm{f}$ ). ICC were also observed in the muscularis mucosa (Fig. 1Bb). Since we used a cryosection, we normally could not observe the whole ICC as an intact shape in one section. c-Kit (+) ICC cells appeared as dark brown ovoid or polyhedral cells as shown in the submucosa and myenteric border (Fig. 1Bb and e).

c-Kit (+) ICC-like immunoreactivity in human gastric mucosa. We found c-Kit (+) ICC-like immunoreactivity in the mucosa. We initially identified structures of gastric mucosa using $H \& E$ staining. The gastric mucosa of the normal fundus/ corpus is composed of fovea and gastric gland (isthmus, neck and base) as shown in Fig. 2A. The isthmus and neck is the 
A

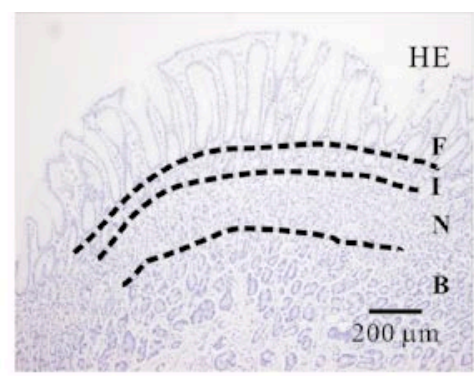

$\mathrm{B}$

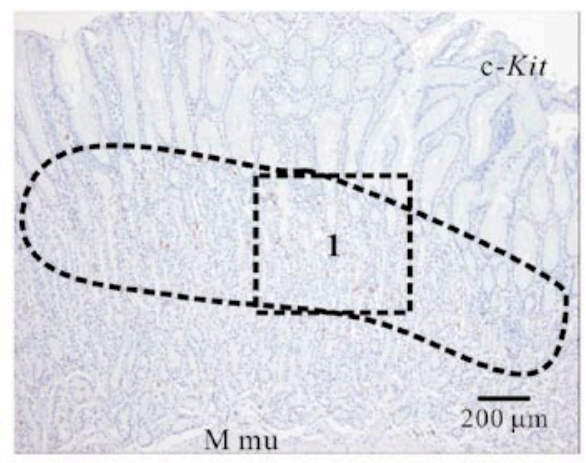

$\mathrm{C}$

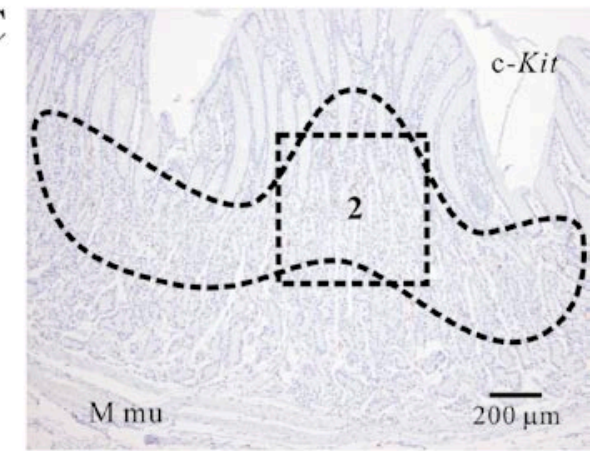

$\mathrm{D}$

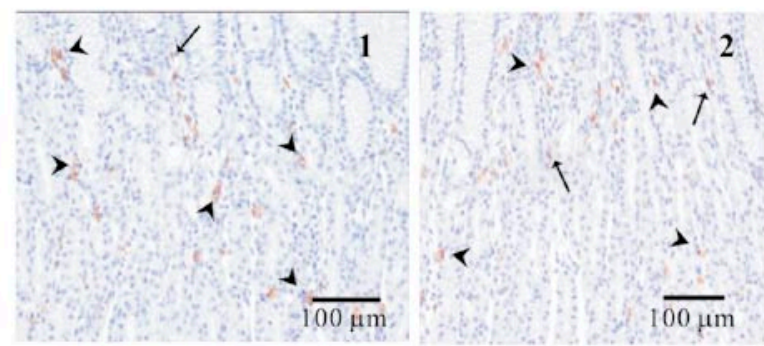

Figure 2. c-Kit (+) ICC-like immunoreactivity in human gastric mucosa. (A) Normal mucosa. F, fovea; I, isthmus; N, neck; B, base. (B-D) Distribution of c-Kit (+) ICC-like cells is in accord with the repair zone. Cell bodies (arrow heads) and processes (arrows) are shown in D.

repair zone (proliferating zone), which contains mucous neck cells. Mucous neck cells are undifferentiated cells that act as stem cells and may migrate upward to renew foveolar and surface epithelium or downward to renew chief, parietal or neuroendocrine cells. As shown in Fig. 2B-D, the distribution of c-Kit (+) ICC-like cells in the normal gastric mucosa was in accord with the repair zone. Their population was very small in normal mucosa which showed no destruction of epithelium by inflammation (Fig. 2B-D). c-Kit (+) ICC-like cells around the base and in the muscularis mucosa appeared to migrate to the repair zone.

c-Kit (+) ICC-like cells in the mucosa with non-metaplastic chronic gastritis without atrophy. Mild/moderate nonmetaplastic chronic gastritis without atrophy is shown in Figs. 3A and 4A. Most of the inflammatory cells were in the superficial mucosa and composed of lymphocytes, plasma cells, eosinophils and neutrophils (Fig. 4B inset 1). Every examined case showed c-Kit (+) ICC-like cells in the mucosa and most of them surrounded the proliferating zone (Figs. 3B and 4C; $n=6)$. They were located close to or attached to epithelia of the proliferating zone (Figs. 3D-F and 4B inset 2). As in the case of ICC present in the gastric muscle (Fig. 1A) (29), c-Kit $(+)$ cells in the mucosa also showed dense cell bodies and processes (Figs. 3D-F and 4B inset 2). Some c-Kit (+) ICC-like cells showed a spindle shape (Fig. 3E and F). A small number of c-Kit (+) ICC-like cells were also found in the base and muscularis mucosa (Fig. 3B).

Epithelium of the repair zone showed weak c-Kit (+) immunoreactivity. Epithelial cells in the proliferating zone exhibited weak c-Kit (+) as shown in Figs. 3D-F, 4C and 4C inset 2 (4B inset 2). Since stromal c-Kit $(+)$ cells and c-Kit $(+)$ epithelial cells were in close approximation with each other, stromal c-Kit $(+)$ cells appeared to undergo mesenchymal epithelial transition (MET) to repair epithelia of the gland. c-Kit $(+)$ immunoreactive cells underwent transition in the epithelia (MET1) and lost their c-Kit immunoreactivity in the cytosol (MET2) then underwent transition to MET3 (Fig. 3F). In other words, it appears that MET2 cells which lose their c-Kit immunoreactivity in the cytosol (M1) do so again at the luminal cell membrane (M2) and finally at the anti-luminal cell membrane.

Patterns of distribution of c-Kit (+) ICC-like cells and mast cells in the mucosa. We studied the distribution of mast cells, another known type of c-Kit $(+)$ cells, in human gastric mucosa. As shown in Fig. 4D, the immnuoreactivity of tryptase $(+)$ mast cells showed different patterns of distribution; it showed even distribution of immunoreactive cells scattered throughout 
A

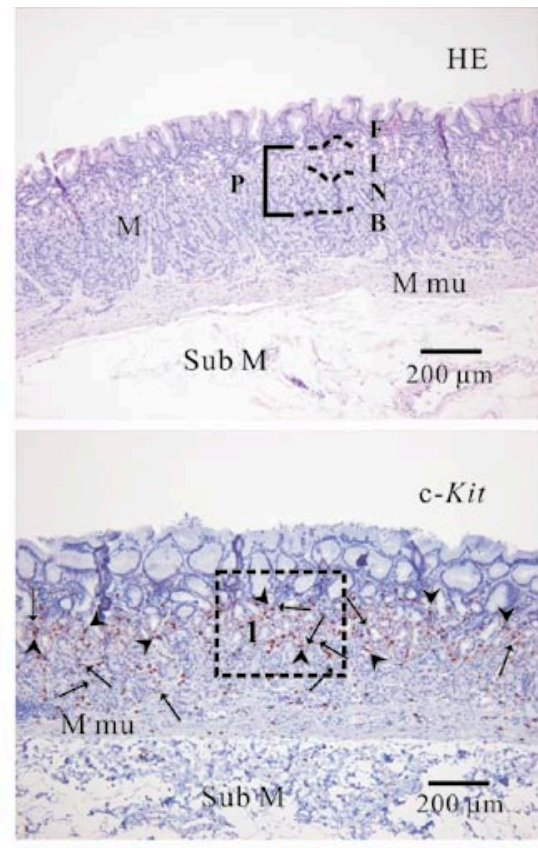

$\mathrm{E}$

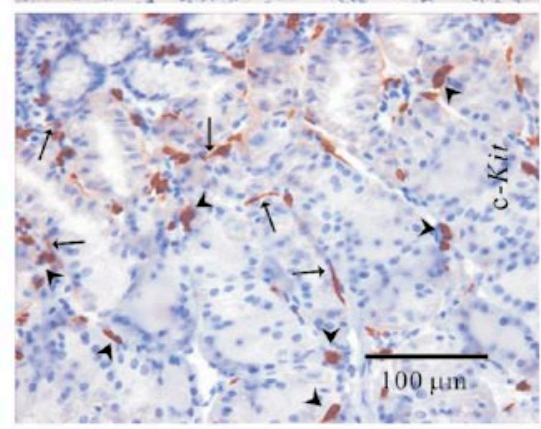

B

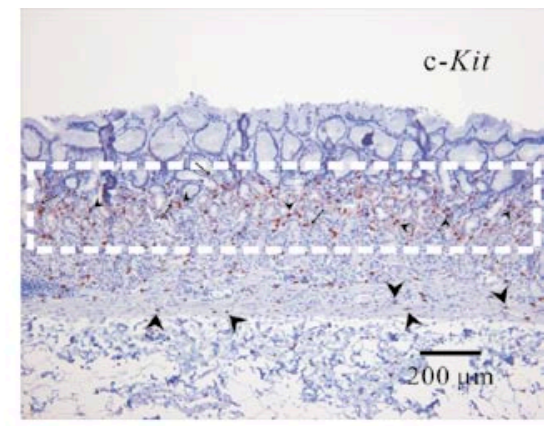

$\mathrm{D}$

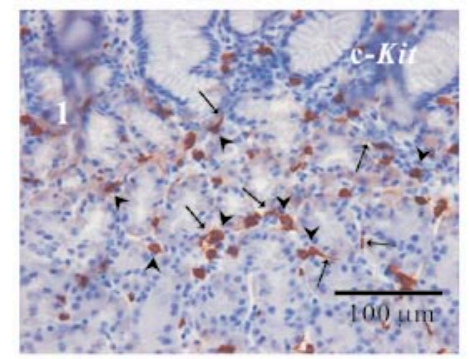

$\mathrm{F}$

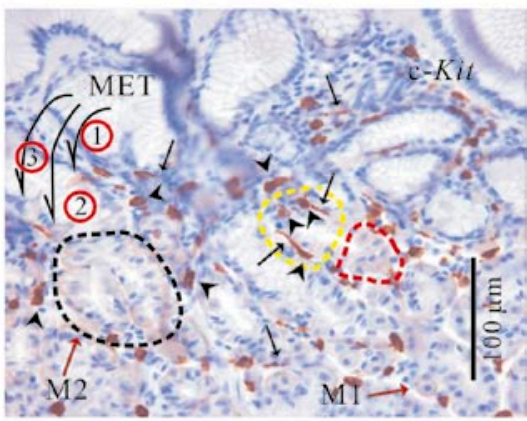

Figure 3. c-Kit (+) ICC-like cells of the mucosa in mild/moderate chronic gastritis without atrophy and intestinal metaplasia. (A) Mild chronic gastritis without atrophy and metaplasia. F, fovea; I, isthmus; N, neck; B, base. c-Kit (+) ICC-like cells were observed mainly around the repair zone (B and C). Epithelial cells of the repair zone also show weak c-Kit-positive immunoreactivity (D-F). Mesenchymal epithelial transition (MET) is observed (F). Cell bodies (arrow heads) and processes (arrows) are shown (D-F); yellow circle in (F).

the whole mucosa, where inflammatory cell infiltration was observed, and showed no confinement to the repair zone (Fig. 4D). This finding indicates that c-Kit (+) ICC-like cells do not originate from mast cells and they are rather associated with the inflammatory reaction.

c-Kit (+) ICC-like cells of the mucosa in non-metaplastic chronic gastritis with (and/or without) atrophy. In severe chronic gastritis with atrophy (Fig. 5A and B), the isthmus and neck of the glands were not distinctive and only some part of the base remained. Mature epithelial cells including parietal cells in isthmus, neck and upper area of the base were destroyed due to severe inflammation. Instead they were replaced by regenerating epithelium. Therefore, the repair zone was expanded to the upper area of the base. In every examined case, we found that the location of c-Kit (+) ICC-like cells matched mostly with the expanded repair zone as in the normal mucosa and nonmetaplastic mucosa with mild/moderate gastritis (Fig. $5 \mathrm{C} ; \mathrm{n}=8$ ).

c-Kit (+) ICC-like cells in lesions of intestinal metaplasia (IM). In non-metaplastic chronic gastritis, c-Kit (+) ICC-like cells were located mainly around the repair zone (Figs. 3B and C, and 4C) and they were obviously attached to epithelial cells
(Figs. 3D-F and 4B inset 2; $\mathrm{n}=8$ ). However, in the presence of IM, c-Kit (+) ICC-like cells showed an evenly scattered distribution in the stroma without confinement to the repair zone (Fig. 6B and D inset 2). Fig. 6A shows chronic gastritis with severe inflammation composed of lymphocytes, plasma cells, eosinophils and neutrophils. The left side of this figure shows an area of moderate atrophy; an expanded repair zone and destroyed structure of the isthmus, neck and upper base. The right side shows a region of IM with severe atrophy; no repair zone and completely destroyed structure of the isthmus, neck and base (Fig. 6A). c-Kit (+) ICC-like cells were found to be attached to epithelia of the repair zone in the atrophic region (Fig. 6B and D inset 1). Meanwhile this phenomenon was rare in IM (Fig. 6B and D inset 2). c-Kit (+) ICC-like cells in IM rather showed an evenly scattered distribution in stroma and did not agglutinate to metaplastic epithelia. In the nonmetaplastic region of the same figure (right side), however, a limited number of repair epithelia were found (Fig. 6B and D inset 3). Therefore, c-Kit (+) ICC-like cells seemed to be scattered evenly in the stroma when the repair zone was replaced by metaplastic epithelium.

The distribution of tryptase-positive mast cells was mainly located where many inflammatory cells existed and did not 
A

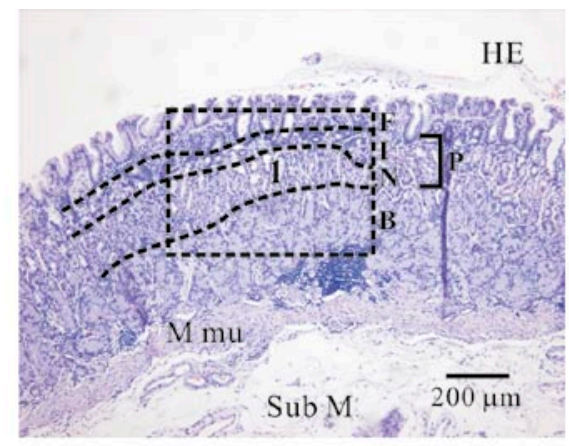

B
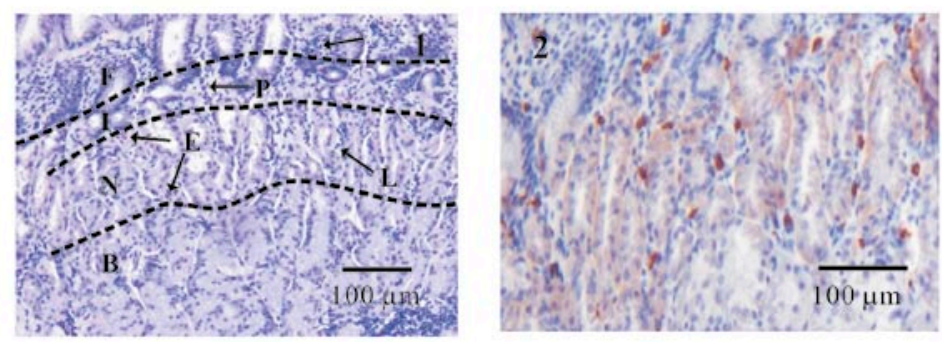

$\mathrm{C}$

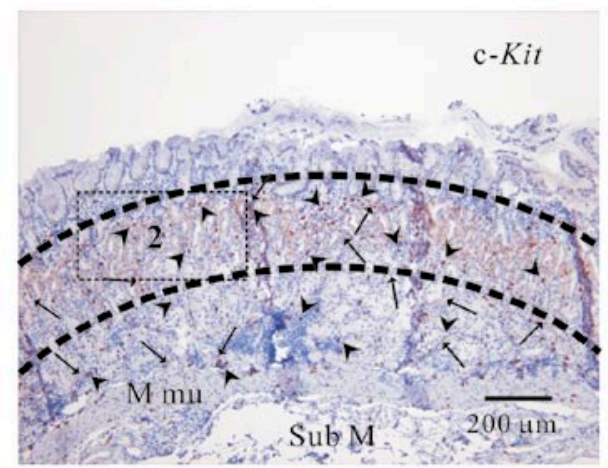

$\mathrm{D}$

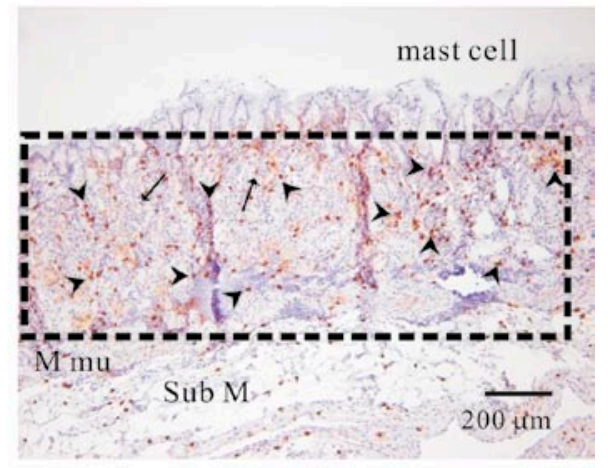

Figure 4. The distribution of c-Kit (+) ICC-like cells, mast cells and inflammatory cells in mild/moderate chronic gastritis without atrophy and intestinal metaplasia. (A) Moderate chronic gastritis (B inset 1). (C) Most c-Kit (+) ICC-like cells were found to be attached to the epithelia of the isthmus and neck of the gastric gland (B inset 2). However, mast cells were scattered evenly in the entire mucosa (D). F, fovea; I, isthmus; N, neck; B, base; L, lymphocyte; E, eosinophil; $\mathrm{P}$, plasma cell.

show any specific distribution pattern associated with the repair zone (Fig. 6C and D inset 4). They were distributed evenly and did not show any correlation with the repair zone in their distribution. In addition, most were found to be close and/or attached to metaplastic epithelium in IM (Fig. 6C and $\mathrm{D}$ inset 4$)$, which was a rare finding in the case of c-Kit (+) ICC-like cells.

c-Kit (+) ICC-like immunoreactivity in gastric adenocarcinoma. The distribution of c-Kit (+) ICC-like cells was also studied in adenocarcinoma using four cases of archival paraffin-embedded tissue of gastric adenocarcinoma. In adenocarcinomatous lesions (Fig. 7A and B inset 1), there was actually no c-Kit (+) ICC-like cells found, although a few cells were found attached to repair epithelia present inside the tumor (Fig. 7A and B inset 1). The adenocarcinoma cells were c-Kit-negative. Thus, it appears that c-Kit (+) ICC-like cells were involved in the regeneration of epithelium. Therefore, it is possible that c-Kit (+) ICC-like cells may be involved in the generation of dysplasia and carcinogenesis by affecting the epithelial regeneration process in human mucosa.

\section{Discussion}

In the present study of the human stomach, we found c-Kit (+) ICC-like cells in the muscle layers including the nerve plexuses and in gastric mucosa. They had the typical morphology of dense fusiform cell bodies with processes and were normally observed at the repair zone in normal mucosa and mucosa of non-metaplastic chronic gastritis. They were usually found adherent to repair epithelium in non-metaplastic chronic gastritis with or without atrophy. Interestingly, these c-Kit (+) ICC-like cells were redistributed relatively evenly over the entire mucosa in the case of chronic gastritis with IM and/or adenocarcinoma. However, mast cells which are also known to exhibit c-Kit positive immunoreactivity showed a different distribution pattern from that of c-Kit $(+)$ ICC-like cells. These findings suggest that c-Kit (+) ICC and/ or ICC-like cells exist in human gastric mucosa and may play an important role in inflammatory mucosa pathology and even in carcinogenesis. This is a novel study on the existence of c-Kit (+) ICC-like cells in the human stomach and on their specific distribution patterns according to the state of mucosa 
A

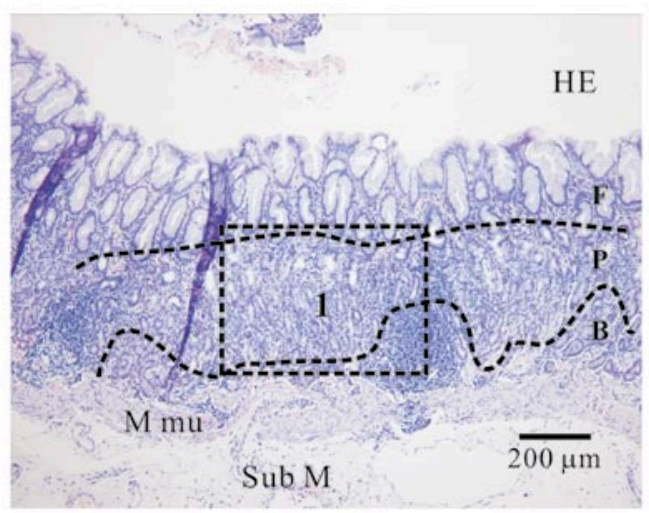

B

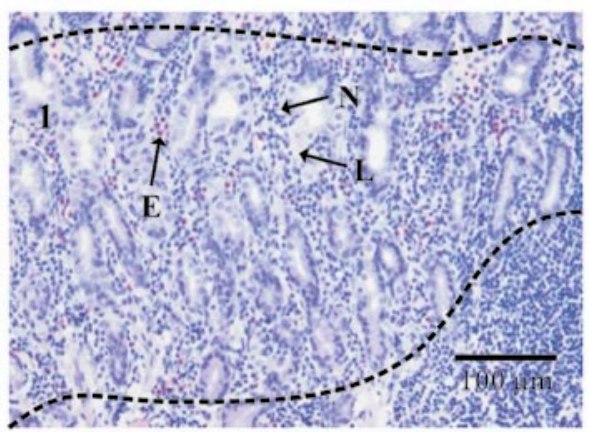

C

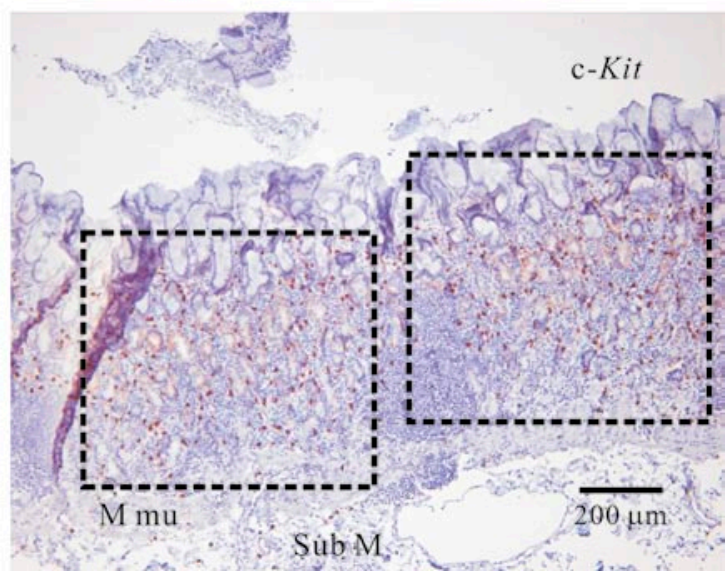

Figure 5. The distribution of c-Kit (+) ICC-like cells and inflammatory severe chronic gastritis with atrophy. In severe chronic gastritis (A and B), atrophy of the gland was observed. We found that the location of c-Kit (+) ICC-like cells matched mostly with the expanded repair zone as in normal mucosa and non-metaplastic mucosa with mild/moderate gastritis (C). F, fovea; B, base; L, lymphocyte; E, eosinophil; P, proliferating (repair) zone; N, neutrophil.

from normal to gastritis with/without atrophy and IM to adenocarcinoma. These results enhance our understanding of the pathophysiology of inflammation and/or carcinogenesis in human gastric mucosa.

As shown in Fig. 1A, we identified c-Kit (+) ICC from human gastric fundus of the greater curvature. ICC were observed throughout all of the layers of the wall of the fundus and corpus in greater and lesser curvature (29). To date, ICC-IM are known to form synaptic contacts with enteric nerve terminals $(1,13)$ and are essential for motor actions to smooth muscle cells $(9,30)$. Although whole morphology was not maintained in the cryosection, we also found ICC-IM in each muscle layer and ICC-SEP in the septa (29). Although not without controversies on the role of ICC (10), ICC are
A

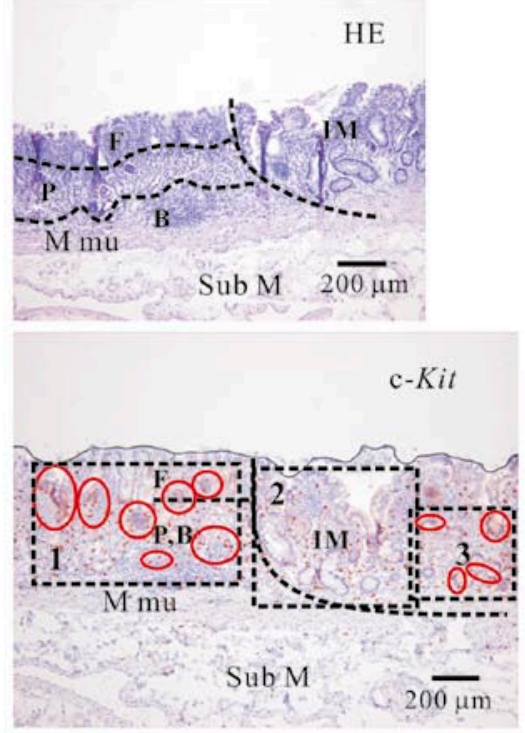

$\mathrm{C}$

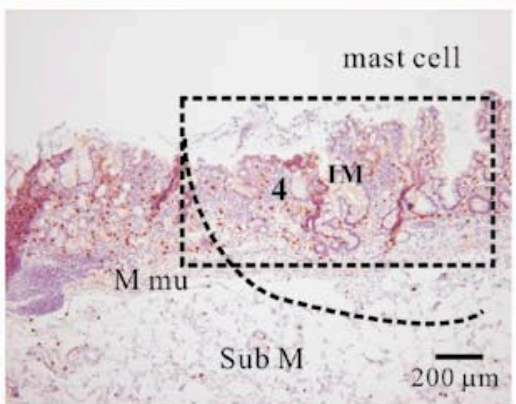

D
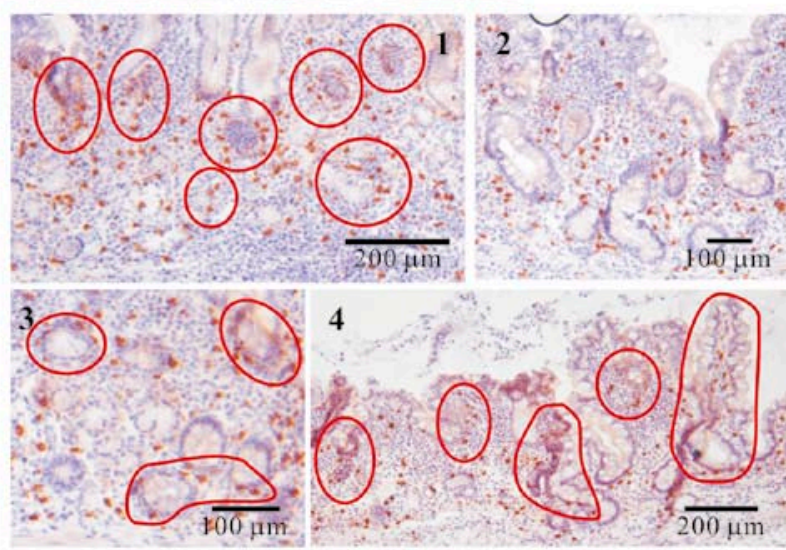

Figure 6. The patterns of the distribution of c-Kit (+) ICC-like cells in severe gastritis with atrophy and in intestinal metaplasia (IM). (A) Chronic gastritis with severe inflammation. The left side of the slide is the area of moderate atrophy and the right side a region of IM with severe atrophy. c-Kit (+) ICClike cells were found attached to epithelia of the repair zone in the atrophic region (B and $\mathrm{D}$ inset 1 ). Meanwhile, these cells in IM were scattered evenly in stroma without agglutination to metaplastic epithelia (B and D inset 2). However, a limited number of repair epithelia remained in the metaplastic region (B and D inset 3). In the atrophic region, tryptase-positive mast cells showed evenly scattered distribution and no association with the repair zone (C and D inset 4). However, many were found closely attached to metaplastic epithelia in the IM region. F, fovea; B, base; P, proliferating (repair) zone.

generally believed to be associated with many GI diseases including motility disorders (11). Furthermore, the loss, damage and hypertrophic change of ICC have been reported in GI tract diseases such as inflammation, viral infection and GIST $(12,25,28,31)$. Thus, further study of ICC is needed to elucidate GI tract disease pathophysiology. 
A

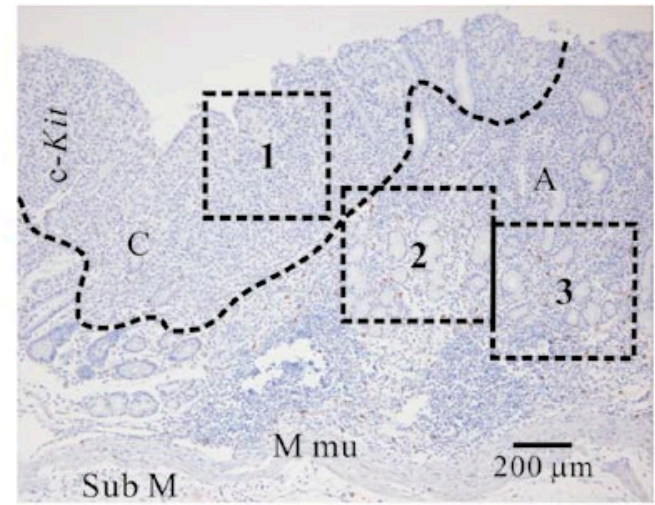

B
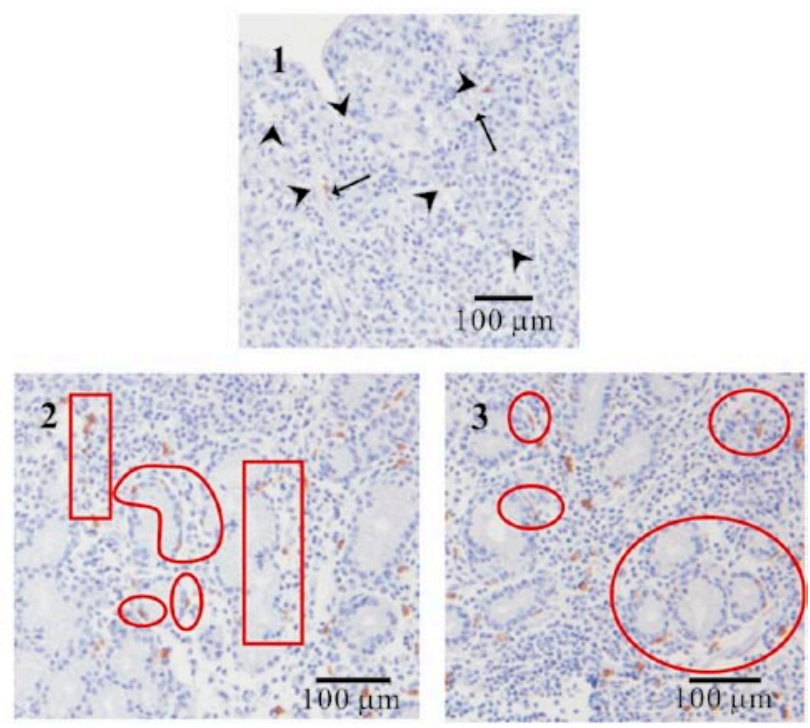

Figure 7. The distribution of c-Kit (+) ICC-like cells in adenocarcinoma. (A) The upper side is the region of signet ring cell adenocarcinoma and the lower side the region of atrophy. Inset 1 of (A) and (B) show the distribution of c-Kit (+) ICC-like cells in adenocarcimoma. A few c-Kit (+) ICC-like cells were found attached to repair epithelia inside the tumor [(B) inset 1]. c-Kit (+) ICC-like cells were seen attached to repair epithelia in the region of atrophy [red circles and boxes in (B) inset 2 and 3]. C, signet ring cell adenocarcinoma; A, atrophy.

ICC normally express c-Kit (RTK), which is essential for survival, proliferation and differentiation of certain cells, for example hematopoiesis, differentiation of mast cells and ICC (32). Therefore, malfunction of c-Kit is thought to be associated with certain neoplastic disorders and gastric dysmotility. Regarding c-Kit-induced malfunction, abnormal c-Kit expression has been observed in a variety of human tumors such as human gastric carcinomas, GIST, small-cell lung cancer and colorectal cancer (17-19). To date, 90 tyrosine kinases have been identified including c-Kit and they are $\gamma$-phosphate groups transferring enzymes from ATP to the hydroxyl group of tyrosine residues on signal transduction molecules and play a central role in signal transduction (33). In addition, some TKs such as c-Kit and epidermal growth factor receptor (EGFR)-TK can be autophosphorylated when activated, as well as phosphorylate other signaling molecules, which leads to dramatic changes in tumor growth (34). Since mutation or overexpression of TK occurs frequently in human malignancies, various TKs have become attractive drug targets for inhibiting cancer. For instance, an inhibitor of TK, imatinib, which acts by competing for occupation of the adenosine triphosphate (ATP)-binding site on the TK domain of the receptor was approved experimentally and is under clinical development (35).

To date, studies on ICC have focused on their pacemaking activity and neuromediation. Only a few studies have focused on the presence of ICC outside the musculature such as in the mucosa, microvilli, submucosal plexus and muscularis mucosa. Notably the brush border of the epithelium in the mucosa of the small intestine was found to express strong immunoreactivity for ICC $(1,13,14,24,29)$. In fact, pathophysiological changes of ICC in the mucosa and submucosa as shown in Figs. 2-7 were reported in gastritis and adenocarcinoma (17). Basically, triggering of the c-Kit receptor by SCF is an essential step for survival and maintenance of the function of ICC. Regarding the role of c-Kit in human GI disease, the aberrant expression of c-Kit was reported in a subgroup of colorectal cancer (19). Furthermore, mutation of c-Kit was associated with some rare human cancers such as GISTs and myeloid leukemia (18). To date, two general mechanisms of c-Kit activation in malignant cells have been described: acquisition of activation mutations and autocrine or paracrine stimulation of the receptor by its ligand SCF (36).

ICC exist everywhere in the GI tract from the esophagus to the internal anus. In addition, their distribution throughout the gastric wall also shows an omnipresence such as ICC-IM, ICC-MY, ICC-SP and ICC in muscularis mucosa and even ICC in mucosa $(1,13,14,24,25,26-40)$. However, the presence and role of ICC outside the muscle layer are seldom studied. However, several studies exist on their role in villi movement, secretion, and tumorigenesis in the mucosa $(17,26,41)$.

In this study, we demonstrated that c-Kit (+) ICC-like cells were found scattered evenly throughout the entire mucosa in severe gastritis ( $n=8$ and adenocarcinoma case) with IM, although they were usually found only in the repair zone in the normal stomach and in non-metaplastic gastritis (Figs. $2 \mathrm{~B}$ and $\mathrm{C}, 3 \mathrm{~B}, 4 \mathrm{C}$ and $6 \mathrm{~B} ; \mathrm{n}=6$ and normal sample). We also demonstrated that $\mathrm{c}-\mathrm{Kit}(+) \mathrm{ICC}$-like cells seemed to adhere to epithelial cells in the repair zone of mucosa (Figs. 3D-F and 4C; $\mathrm{n}=6$ ). Furthermore, as shown in Fig. 3F, they appeared to exhibit MET ability, since epithelial cells showed weak c-Kit-positive immunoreactivity, and c-Kit (+) ICC-like cells showed a stepwise loss of c-Kit-positive immunoreactivity (MET1-3. M1-M2 cells, Fig. 3F). These results imply the involvement of c-Kit (+) ICC-like cells in the repair of epithelium. In fact, epithelial repair would not be prominent in normal mucosa, compared with that of inflammatory mucosa, and malfunction of the repair process in chronic gastritis could promote dysplasia and even carcinogenesis.

c-Kit was found to be expressed in a gastric carcinoma cell line (17). Our study demonstrated further that c-Kit (+) ICC-like cells showed different patterns of distribution according to disease state and showed a close relation with repair epithelium. These results imply a possible role in inflammatory mucosa pathology and carcinogenesis in the human stomach. However, we do not know whether mutated c-Kit activation is involved independently of SCF at this moment.

In the stomach, several risk factors for tumorigenesis of adenocarcinomas were reported: $H$. pylori infection as a class I carcinogen and stress, genetic mutation, and salty food intake. Gastric cancer also exhibits much regional and 
personal dependency on diet especially in Asian countries. At least 8 of the studied tissue samples were $H$. pylori-positive. However, we cannot explain the relation between $H$. pylori infection and pathology of c-Kit (+) ICC-like cells of mucosa, and plan to further investigate this possibility.

In gastric mucosa, in general, intestinal-type gastric cancer typically arises in the setting of chronic gastritis and develops through intermediate stages of atrophic gastritis, IM, dysplasia, and finally gastric cancer. This lengthy process is dependent on continued chronic inflammation (42). Metaplasia is a particularly significant feature as it is a fairly permanent alteration that suggests a marked change in the genetic and epigenetic program of the gastric stem or progenitor cells. In general, chronic inflammation induces increased tissue turnover, which is thought to predispose to an excessive rate of proliferation, and in many cases results in more frequent mitotic errors and an increased rate of mutagenesis. In addition, cancer arises from cancer stem cells (CSCs) and the emergence of CSCs by chronic inflammation needs to be addressed. Gastric stem, progenitor cells and/or bone marrow-derived cells (BMDCs) may also be good candidates for gastric CSCs (21). Previously, strong evidence that gastric cancer can originate from bone-marrow-derived sources and transformation of stem cells (21) was reported. Surprisingly, BMDCs possess a wide range of plasticity, migrating ability through peripheral organs; therefore, they may contribute directly or indirectly to epithelial cancer, as a result of inflammation and tissue injury. Notably, ICC which exhibit plasticity are one type of MSCs and development is dependent on c-Kit activation (22).

Furthermore, motility disorder by ethanol-induced inflammation and Trichinella spiralis infection in the GI tract is also known to be due to injury of ICC, resulting in reduction of density, damage of ICC processes and vacuolization of their cytoplasm $(25,28)$. These characteristics and findings suggest that ICC are associated with carcinogenesis in the mucosa. In fact, gastric epithelium at the repair zone of the mucosa showed weak c-Kit-positive immunoreactivity as shown in Figs. 3D-F and 4B. In addition, it seems that c-Kit (+) ICC-like cells may have MET ability to epithelium. Also, c-Kit (+) ICC-like cells in human gastric mucosa were found to be attached to repair epithelium and increased numbers we found in gastritis and atrophy compared to normal tissue (Figs. 2B and C, 3B, 4C and $5 \mathrm{C}$ ). The most important point of this study is that $\mathrm{c}-\mathrm{Kit}(+)$ ICC-like cells were observed, and the distribution was associated to repair epithelium in case of non-metaplastic gastritis with or without atrophy. In addition, they were located close to epithelial cells in those cases although they did not invaginate to them (Figs. 3D-F and 4B). As mentioned above, inflammation was known to stimulate their growth and enhance their further progression to cancer, and chronic inflammation induces increased tissue turnover and causes more frequent mitotic errors and an increased rate of mutagenesis. Therefore, among the many reported mechanisms of carcinogenesis, it seems that the expression and particular distribution of c-Kit $(+)$ ICC-like cells, one type of MSCs, may have tumor-promoting and/or proliferating abilities associated with chronic gastritis in the human stomach (17).

As shown in Figs. 2B and C, 3B and C, and 4C of normal, mild and moderate gastritis, c-Kit (+) ICC-like cells showed migratory ability to the repair zone from the propria of mucosa. Furthermore, c-Kit (+) ICC-like cells were redistributed in whole gastric mucosa associated with intestinal metaplasia in which normal repair zone is lost. Unfortunately, we cannot explain this phenomenon, however, the close approximation of epithelium and c-Kit (+) ICC-like cells which show a typical phenotype of ICC such as side branches from dense cell bodies may suggest an important role for inflammatory-induced changes in mucosa (Fig. 3C-F) $(25,28)$.

\section{References}

1. Cajal SR: Histologie du systèm nerveux de l'homme et des vertébrés. 2nd edition, Maloine, Paris, pp891-942, 1911.

2. Huizinga JD, Thuneberg L, Kluppel M, et al: W/Kit gene required for interstitial cells of Cajal and for intestinal pacemaker activity. Nature 373: 347-349, 1995.

3. Torihashi S, Ward SM and Sanders KM: Development of c-Kitpositive cells and the onset of electrical rhythmicity in murine small intestine. Gastroenterology 112: 144-155, 1997.

4. Radenkovic G, Savic V, Mitic D, Grahovac S, Bjelakovic M and Krstic M: Development of c-Kit immunopositive cells of Cajal in the human stomach. J Cell Mol Med 14: 1125-1134, 2010.

5. Faussone-Pellegrini MS and Cortesini C: Ultrastructural features and localization of the interstitial cells of Cajal in the smooth muscle coat of human esophagus. J Submicrosc Cytol 17: 187-197, 1985.

6. Romert P and Mikkelsen HB: c-Kit immunoreactive cells of Cajal in the human small and large intestine. Histochem Cell Biol 109: 195-202, 1998.

7. Hagger R, Gharaie S, Finlayson C, et al: Regional and transmural density of interstitial cells of Cajal in human colon and rectum. Am J Physiol 275: G1309-G1316, 1998.

8. Burns AJ, Herbert TM, Ward SM, et al: Interstitial cells of Cajal in the guinea-pig gastrointestinal tract as revealed by c-Kit immunohistochemistry. Cell Tissue Res 290: 11-20, 1997.

9. Burns AJ, Lomax AE, Torihashi S, et al: Interstitial cells of Cajal mediated inhibitory neurotransmission in the stomach. Proc Natl Acad Sci USA 93: 12008-12013, 1996.

10. Goyal RK and Chaudhury A: Mounting evidence against the role of ICC in neurotransmission to smooth muscle in the gut. Am J Physiol 298: G10-G13, 2009.

11. Vanderwinden JM and Rumessen JJ: Interstitial cells of Cajal in human gut and gastrointestinal disease. Microsc Res Tech 47: 344-360, 1999.

12. Hirota S, Okazaki T, Kitamura Y, et al: Cause of familial and multiple gastrointestinal autonomic nerve tumors with hyperplasia of interstitial cells of Cajal is germline mutation of the c-Kit gene. Am J Surg Pathol 24: 326-327, 2000.

13. Güldner FH, Wolff DG and Keyserlingk DG: Fibroblasts as a part of the contractile system in duodenal villi of rat. Z Zellforsch Mikrosk Anat 135: 349-360, 1972.

14. Torihashi S, Yokoi K, Nagaya H, et al: New monoclonal antibody (AIC) identifies interstitial cells of Cajal in the musculature of the mouse gastrointestinal tract. Auton Neurosci 113: 16-23, 2004.

15. Klüppel M, Huizinga JD, Malysz J, et al: Developmental origin and Kit-dependent development of the interstitial cells of Cajal in the mammalian small intestine. Dev Dyn 211: 60-71, 1998.

16. Rygaard K, Nakamura T and Spang Thomsen M: Expression of the proto-oncogene $c$-met and c-Kit and their ligands, hepatocyte growth factor/scatter facter and stem cell factor, in SCLS cell lines and xenografts. Br J Cancer 67: 37-46, 1993.

17. Hassan S, Kinoshita Y, Kawanami C, et al: Expression of protooncogene c-Kit and its ligand stem cell factor (SCF) in gastric carcinoma cell lines. Dig Dis Sci 43: 8-14, 1998.

18. Feng F, Liu XH, Xie Q, et al: Expression and mutation of c-Kit gene in gastrointestinal stromal tumors. World J Gastroenterol 9: 2548-2551, 2003.

19. Bellone G, Smirne C, Carbone A, et al: KIT/stem cell factor expression in premalignant and malignant lesions of the colon mucosa in relationship to disease progression and outcomes. Int J Oncol 29: 851-859, 2006.

20. Piazuelo MB, Camargo MC, Mera RM, et al: Eosinophils and mast cells in chronic gastritis: possible implications in carcinogenesis. Hum Pathol 39: 1360-1369, 2008. 
21. Houghton J, Stoicov C, Nomura S, et al: Gastric cancer originating from bone marrow-derived cells. Science 306: 1568-1571, 2004.

22. Torihashi S, Nishi K, Tokutomi Y, et al: Blockade of kit signaling induces transdifferentiation of interstitial cells of Cajal to a smooth muscle phenotype. Gastroenterology 117: 140-148, 1999.

23. Fujiwara $\mathrm{T}$, Motoyama $\mathrm{T}$, Ishihara $\mathrm{N}$, et al: Characterization of four new cell lines derived from small-cell gastrointestinal carcinoma. Int J Cancer 54: 965-971, 1993.

24. Kunisawa $Y$ and Komuro T: Interstitial cells of Cajal associated with submucosal plexus of the guinea-pig stomach. Neurosci Lett 434: 273-276, 2008.

25. Popescu LM, Gherghiceanu M, Cretoiu D, et al: The connective connection: interstitial cells of Cajal-like cells establish synapses with immunoreactive cells. J Cell Mol Med 9: 714-730, 2005.

26. Percy WH, Warren JM and Brunz JT: Characteristics of the muscularis mucosae in the acid-secreting region of the rabbit stomach. Am J Physiol 276: G1213-G1220, 1999.

27. Fawcett DW: A Textbook of Histology. 12th edition, Chapman \& Hall, New York, pp617-651, 1994.

28. Der T, Bercik P, Donnelly G, et al: Interstitial cells of Cajal and inflammation-induced motor dysfunction in the mouse small intestine. Gastroenterology 119: 1590-1599, 2000.

29. Yun HY, Sung RH, Kim YC, et al: Regional distribution of interstitial cells of Cajal (ICC) in human gastric stomach. Korean J Physiol Pharmacol 14: 317-324, 2010.

30. Wang XY, Paterson $C$ and Huizinga JD: Cholinergic and nitrergic innervation of ICC-DMP and ICC-IM in the human small intestine. Neurogastroenterol Motil 15: 531-543, 2003.

31. Oh JJ and Kim CH: Gastroparesis after a presumed viral illness: clinical and laboratory features and natural history. Mayo Clin Proc 65: 636-642, 1990.
32. Ashiman LK: The biology of stem cell factor and its receptor c-Kit. Int J Biochem Cell Biol 31: 1037-1051, 1999.

33. Schlessinger J: Cell signaling by receptor tyrosine kinases. Cell 103: 211-225, 2000.

34. Olayioye MA, Neve RM, Lane HA, et al: The ErbB signaling network: receptor heterodimerization in development and cancer. EMBO J 19: 3159-3167, 2000.

35. Buchdunger E, Cioffi CL, Law N, et al: Abl protein-tyrosine kinase inhibitor STI571 inhibits in vitro signal transduction mediated by c-Kit and platelet-derived growth factor receptors. J Pharmacol Exp Ther 295: 139-145, 2000.

36. Vlahovic $\mathrm{G}$ and Crawford J: Activation of tyrosine kinases in cancer. Oncologist 8: 531-538, 2003.

37. Rumessen JJ and Thunebereg L: Pacemaker cells in the gastrointestinal tract: interstitial cells of Cajal. Scan J Gastroenterol 216: 82-94, 1996.

38. Ibba Manneschi L, Pacini S, Corsani L, et al: Interstitial cells of Cajal in the human stomach: distribution and relationship with enteric innervation. Histol Histopathol 19: 1153-1164, 2004.

39. Nemeth L, Maddeur S and Puri P: Immunolocalization of the gap junction protein connexin 43 in the interstitial cells of Cajal in the normal and Hirschsprung's disease bowel. J Pediatr Surg 35: 823-828, 2000.

40. Nemeth L and Puri P: Three-demensional morphology of c-Kitpositive cellular network and nitrergic innervation in the human gut. Arch Pathol Lab Med 125: 899-904, 2001.

41. Synnerstad I, Ekblad E, Sundler F, et al: Gastric mucosal smooth muscles may explain oscillations in glandular pressure: role of vasocative intestinal peptide. Gastroenterology 114: 284-294, 1998.

42. Correa P and Houghton J: Carcinogenesis of Helicobacter pylori. Gastroenterology 133: 659-672, 2007. 